\title{
Survival effects of a strategy favoring second-line multimodal treatment compared to supportive care in glioblastoma patients at first progression
}

\author{
Pantelis Stavrinou, MD, PhD, ${ }^{1}$ Aristotelis Kalyvas, MD, ${ }^{2}$ Stefan Grau, MD, ${ }^{1}$ Christina Hamisch, MD, \\ Norbert Galldiks, MD, ${ }^{3-5}$ Sotirios Katsigiannis, MD, ${ }^{1}$ Christoph Kabbasch, MD, ${ }^{6}$ \\ Marco Timmer, MD, ${ }^{1}$ Roland Goldbrunner, MD, ${ }^{1}$ and George Stranjalis, MD² \\ 1'Department of Neurosurgery, University Hospital of Cologne, Germany; 2Department of Neurosurgery, Evangelismos Hospital, \\ University of Athens, Greece; ${ }^{3}$ Department of Neurology, University Hospital Cologne, Germany; ${ }^{4}$ nstitute of Neuroscience and \\ Medicine, Research Center Juelich, Germany; ${ }^{5}$ Center of Integrated Oncology, Universities of Cologne and Bonn, Cologne, \\ Germany; and ${ }^{6}$ Department of Neuroradiology, University Hospital Cologne, Germany
}

OBJECTIVE Data on the survival effects of supportive care compared to second-line multimodal treatment for glioblastoma progression are scarce. Thus, the authors assessed survival in two population-based, similar cohorts from two European university hospitals with different treatment strategies at first progression.

METHODS The authors retrospectively identified patients with newly diagnosed glioblastoma treated at two neurooncological centers. After diagnosis, patients from both centers received identical treatments, but at tumor progression each center used a different approach. In the majority of cases, at center A (Greece), supportive care or a single therapeutic modality was offered at progression, whereas center B (Germany) provided multimodal second-line therapy. The main outcome measure was survival after progression (SaP). The influence of the treatment strategy on SaP was assessed by multivariate analysis.

RESULTS One hundred three patients from center $A$ and 156 from center $B$ were included. Tumor progression was observed in 86 patients (center A) and 136 patients (center B). At center A, 53 patients (72.6\%) received supportive care alone, while at center B, 91 patients $(80.5 \%)$ received second-line treatment. Progression-free survival at both centers was similar ( 9.4 months [center $A$ ] vs 9.0 months [center $B$ ]; $p=0.97)$, but SaP was significantly improved in the patients treated with multimodal second-line therapy at center $\mathrm{B}$ (7 months, $95 \% \mathrm{Cl} 5.3-8.7$ months) compared to those treated with supportive care or a single therapeutic modality at center A (4.5 months, 95\% Cl 3.5-5.5 months; $p=0.003$ ). In the multivariate analysis, the treatment center was an independent prognostic factor for overall survival $(\mathrm{HR} 1.59,95 \% \mathrm{Cl}$ $0.17-2.15 ; p=0.002)$.

CONCLUSIONS Treatment strategy favoring multimodal second-line treatment over minimal treatment or supportive care at glioblastoma progression is associated with significantly better overall survival.

https://thejns.org/doi/abs/10.3171/2018.7.JNS18228

KEYWORDS glioblastoma; palliative care; progression; second-line treatment; survival; therapy; oncology

$\mathrm{G}$ LIOBLASTOMA (GBM) is the most common primary malignant brain tumor in adults, with an incidence of 5-6 new cases per 100,000 individuals per year worldwide. ${ }^{27}$ Despite continuous research into the treatment of GBM, prognosis remains dismal, with a current median survival between 15 and 17 months., ${ }^{2,22,23}$ Prior to 2005, first-line treatment of GBM was not standardized, with the implementation of chemotherapy being only cir- cumstantial. ${ }^{9}$ In 2005, the results of the European Organisation for Research and Treatment of Cancer (EORTC) 22981/26981 trial showed that radiotherapy plus concomitant and adjuvant temozolomide-based chemotherapy improves progression-free survival (PFS) and overall survival (OS) compared to radiotherapy alone, making this treatment the standard of care for newly diagnosed GBMs. ${ }^{22}$

Nevertheless, GBM progression occurs in almost all

ABBREVIATIONS EORTC = European Organisation for Research and Treatment of Cancer; GBM = glioblastoma; KPS = Karnofsky Performance Scale; $M G M T=$ $0^{6}$-methylguanine-DNA methyltransferase; OS = overall survival; $\mathrm{PFS}=$ progression-free survival; $\mathrm{QOL}$ = quality of life; $\mathrm{SaP}=$ survival after progression. SUBMITTED January 25, 2018. ACCEPTED July 3, 2018.

INCLUDE WHEN CITING Published online November 30, 2018; DOI: 10.3171/2018.7.JNS18228. 
TABLE 1. Baseline and treatment characteristics

\begin{tabular}{|c|c|c|c|}
\hline \multirow[b]{2}{*}{ Characteristic } & \multicolumn{2}{|c|}{ Value } & \multirow[b]{2}{*}{$\begin{array}{c}p \\
\text { Value }\end{array}$} \\
\hline & $\begin{array}{l}\text { Center A } \\
(n=103)\end{array}$ & $\begin{array}{l}\text { Center B } \\
(n=156)\end{array}$ & \\
\hline Mean age (SD), yrs & $59.3(11.9)$ & $59.1(12.2)$ & 0.88 \\
\hline Men & $63(61.2)$ & $98(62.8)$ & 0.79 \\
\hline KPS score $\geq 70$ & $91(88.3)$ & $146(93.6)$ & 0.14 \\
\hline IDH mutation & $3(5.1)^{*}$ & $5(7.0)^{*}$ & 0.16 \\
\hline Midline involvement & $16(15.5)$ & $21(13.5)$ & 0.64 \\
\hline Left side & $58(56.3)$ & $78(50)$ & 0.32 \\
\hline Median hospital stay (SD), days & $8(8.5)$ & $8(4.8)$ & 0.52 \\
\hline Patients with progression & $86(83.5)$ & $136(87.2)$ & \\
\hline Median duration of symptoms, wks & 4 & 4 & 0.84 \\
\hline \multicolumn{4}{|l|}{ 2nd-line treatment } \\
\hline Any & $20(23.2)$ & $91(67)$ & \multirow{3}{*}{$<0.001$} \\
\hline Supportive care & $53(61.6)$ & $22(16.2)$ & \\
\hline Data missing & $13(15.1)$ & $23(16.9)$ & \\
\hline $\begin{array}{l}\text { Values are presented as the number }\left({ }^{\circ}\right. \\
\text { (SD). } \\
{ }^{*} \text { The presence of an IDH mutation wa } \\
\text { A and } 71 \text { patients in center B. The per } \\
\text { whom IDH status was determined. }\end{array}$ & ermin & patien & $\begin{array}{l}\text { dian } \\
\text { ther } \\
\text { ts in }\end{array}$ \\
\hline
\end{tabular}

patients, and, unlike the uniform first-line treatment, no standard-of-care treatment has been established for recurrent disease, as no treatment strategy has been shown to conclusively improve outcome. ${ }^{26}$ At first progression, therapeutic approaches frequently involve a combination of resection, reirradiation, and alkylating chemotherapy. However, evidence for the efficacy of these therapies is rather limited and inconclusive. . $, 7,16,18$

Furthermore, it is still unclear whether second-line treatment of GBM at first progression achieves better survival than supportive care alone. The very few studies that address this question usually compare cohorts generated from a single institution after certain selection criteria are met (based on competing comorbidities, performance status, or resectability of the tumor at baseline) or compare cohorts from before and after the Stupp era. ${ }^{5,14,15,20}$ In the former, there is a profound selection bias, and in the latter, no sure conclusion can be drawn due to the fact that the effect of the second-line therapy cannot be isolated from that of the primary treatment. This leads to a great discrepancy between applied practices, which ultimately are governed by various factors such as the treating physician's preference, regional nonvalidated protocols, availability of specialized equipment (such as that for stereotactic radiotherapy, radiosurgery, or brachytherapy), and the structure and financial resources of the health system itself.

We conducted an analysis of two very similar cohorts of GBM patients treated in neurooncological centers in Greece and in Germany. In both countries, maximal safe tumor resection followed by a treatment according to the EORTC 22981/26981 trial is provided; however, their treatment strategies at disease progression are very different. Neurooncological centers in Germany frequently favor an interdisciplinary approach and usually offer spe- cific treatment at first progression..$^{20}$ In contrast, patients in Greece are typically managed outside of an interdisciplinary setting, by either the operating neurosurgeon or a general oncologist. At first progression, supportive care alone is usually offered, with only a minority of patients receiving second-line treatment.

The purpose of this study was to examine whether a strategy favoring active treatment of GBM at progression offers an advantage in OS compared to supportive care alone.

\section{Methods}

We retrospectively analyzed two newly diagnosed GBM patient cohorts that were treated between June 2010 and June 2015 at the Department of Neurosurgery, Evangelismos Hospital, University of Athens, Greece (center A), and Neurooncological Center, University Hospital of Cologne, Germany (center B). Medical records were reviewed to identify adult patients with newly diagnosed primary GBM who were eligible for treatment according to the EORTC 22981/26981 protocol. Neuropathological diagnosis was performed in both cohorts by an experienced neuropathologist in accordance with the WHO classification of 2007. ${ }^{12}$ Patients with infratentorial, multilocular, and secondary GBM, patients who received only biopsy or underwent tumor-debulking surgery, and patients not suitable for Stupp protocol treatment were excluded.

Medical records were reviewed for clinical variables such as sex, age at diagnosis, Karnofsky Performance Scale (KPS) score documented at admission and at each follow-up visit, location of the tumor, time from symptom onset to diagnosis, presenting symptoms, days of hospital stay, and second-line treatment. Baseline characteristics are summarized in Table 1.

In both cohorts, the tumor resection was performed by experienced neurosurgeons using a state-of-the-art neurosurgical microscope (Pentero OPMI, Karl Zeiss), navigation (StealthStation [Medronic] at center $\mathrm{A}$ and VectorVision [BrainLab] at center B), and intraoperative electrophysiological monitoring. Postoperative contrastenhanced MR images were obtained no later than 48 hours after surgery.

Follow-up comprised clinical examination and MRI every 3 months or upon clinical deterioration. Tumor recurrence was defined using the RANO criteria..$^{29}$ In the case of suspected tumor progression, patients were reassessed by MRI after 6 weeks.

The primary endpoint was survival after progression $(\mathrm{SaP})$ defined as time from progression to death or the end of follow-up (December 2016 for both cohorts). PFS was defined as time from first surgery to first progression.

In the case of tumor progression, the further course of action at center A was determined solely by the treating physician, either a neurosurgeon or an oncologist. At center B, patients' cases were discussed by members of an interdisciplinary tumor board.

\section{Statistical Analysis}

Quantitative baseline patient characteristics were compared using the Student t-test or Mann-Whitney U-test in 
TABLE 2. Treatment modalities for patients with recurrence

\begin{tabular}{ccc}
\hline \multirow{2}{*}{ 2nd-Line Treatment } & \multicolumn{2}{c}{ No. of Patients $(\%)$} \\
\cline { 2 - 3 } & Center A $(n=73)^{*}$ & Center B $(n=113)^{*}$ \\
\hline Monotherapy & $15(20.5)$ & $33(29.2)$ \\
\hline Op & $11(15)$ & $12(10.6)$ \\
\hline CTX & $1(1.4)$ & $20(17.7)$ \\
\hline Rx & $3(4.1)$ & $1(0.9)$ \\
\hline Combination treatment & $5(6.8)$ & $58(51.3)$ \\
\hline Op + Rx & & $5(4.4)$ \\
\hline Op + CTX & & $10(8.8)$ \\
\hline Op + Rx + CTX & & $16(14.2)$ \\
\hline Op + CTX + Bev & & $5(4.4)$ \\
\hline Op + Rx + CTX + Bev & & $4(3.5)$ \\
\hline Op + Bev & & $2(1.8)$ \\
\hline Rx + CTX & & $2(1.8)$ \\
\hline Rx + Bev & & $1(0.9)$ \\
\hline Rx + CTX + Bev & & $1(0.9)$ \\
\hline CTX + Bev & $1(1.4)$ & $11(9.7)$ \\
\hline Bev & $4(5.5)$ & $1(0.9)$ \\
\hline Supportive care & $53(72.6)$ & $22(19.5)$ \\
\hline
\end{tabular}

Bev = bevacizumab; $\mathrm{CTX}=$ chemotherapy; $\mathrm{Rx}=$ radiotherapy.

* Missing cases excluded (center A: 13 [15\%] missing cases and center B: 23 $[17 \%] ; p=0.30)$.

the case of violation of normality. Categorical variables are reported as counts/numbers and percentages and differences between the cohorts were evaluated using Pearson's chi-square or Fisher's exact test, as appropriate. SaP and PFS were estimated using the Kaplan-Meier analysis, and the log-rank test was used for group comparisons. A Cox proportional hazards regression model was used for univariate and multivariate analyses to test the effect of prognostic factors in terms of SaP. A two-tailed $p$ value of $\leq 0.05$ was considered statistically significant. All statistical analyses were performed using SPSS version 20.0 (IBM Corp.).

For this type of study, no ethical approval was required.

\section{Results \\ Patients}

A total of 431 GBM patients treated within the study period could be identified. Inclusion criteria were not met by 28 patients from center A (12 with multilocular tumors, 9 who underwent tumor debulking, 5 with infratentorial lesions, and 2 who refused treatment) and 38 patients from center B (16 with multilocular tumors, 12 who underwent tumor debulking, 9 with infratentorial lesions, and 1 who refused treatment), and 106 were excluded because the exact point of progression or the OS could not be established. In total, 103 patients treated in center A and 156 patients treated in center B were included in this analysis. Both groups were very similar in terms of age at diagnosis, male/female ratio, duration of symptoms, KPS score, and the presence of an IDH mutation. IDH mutations were diagnosed in 59 patients $(57 \%)$ at center $\mathrm{A}$ and 71 patients

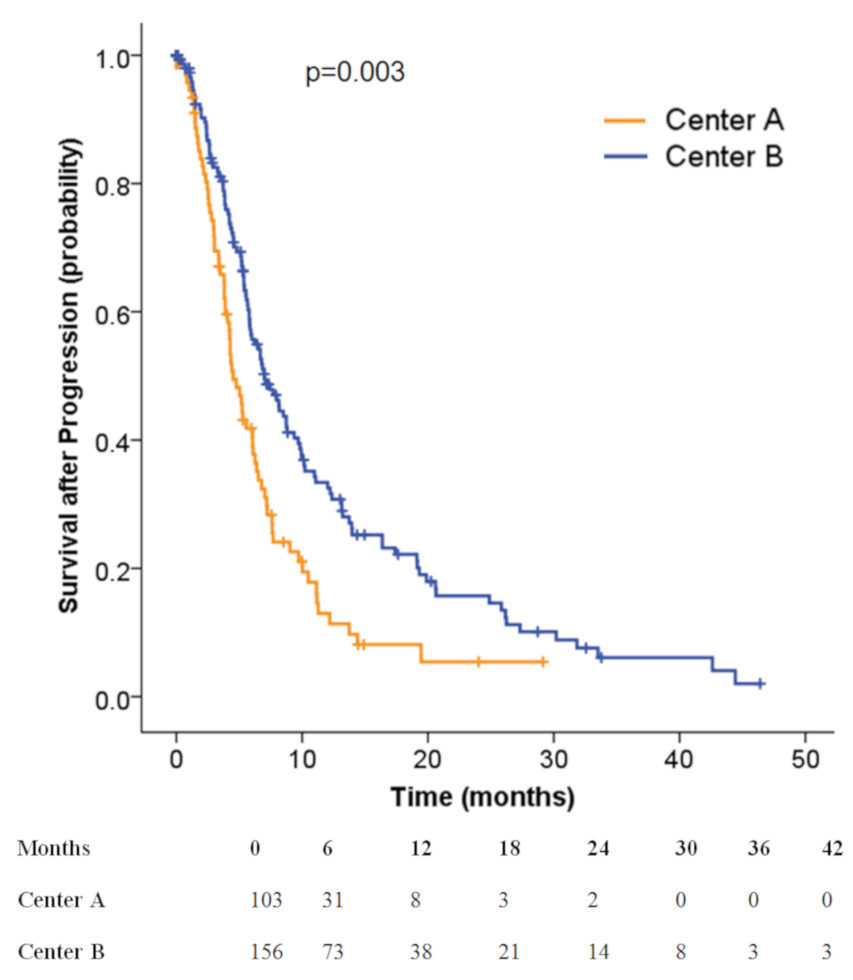

FIG. 1. Kaplan-Meier curve for SaP between center A and center B, showing significantly longer survival for patients treated in center $B(p=$ 0.003). Hash marks indicate censored cases.

$(45.5 \%)$ at center B. The implementation of intraoperative aiding tools was similar in both departments; navigation was used in $72 \%$ and $67 \%$ of cases a centers $A$ and $\mathrm{B}$, respectively, while intraoperative electrophysiological monitoring was used in $53 \%$ of patients from center A and $21 \%$ from center B (difference not significant). For both cohorts, median hospital stay after resection was 8 days (Table 1).

The two cohorts did not differ significantly regarding permanent postoperative deficits $(7 \%$ vs $18 \%$ in center A and center $\mathrm{B}$, respectively; $\mathrm{p}=0.21)$. For $26(10 \%)$ patients, the exact number of adjuvant temozolomide cycles was not documented. For the remaining patients, $55 \%$ in center A and $48.1 \%$ in center B could complete the 6 cycles of adjuvant temozolomide treatment $(\mathrm{p}=0.30)$.

For 13 of the 86 patients whose tumors progressed in center A and 23 of 136 in center B, the further course of treatment is unknown. For the rest of the patients, there was a significant discrepancy between the favored strategy after disease progression or recurrence, with $27.4 \%$ of patients treated in center A receiving further treatment, as opposed to $80.5 \%$ of patients in center B ( $p<0.001)$ (Table 2).

\section{Survival}

$\mathrm{SaP}$ was significantly longer for patients treated in center B (7 months, 95\% CI 5.3-8.7 months) than for patients treated in center A (4.5 months, 95\% CI 3.5-5.5 months; $\mathrm{p}=0.003)$ (Fig. 1).

The estimated survival rate at 3 months after progression was $69 \%$ for center A patients compared with $82.5 \%$ 


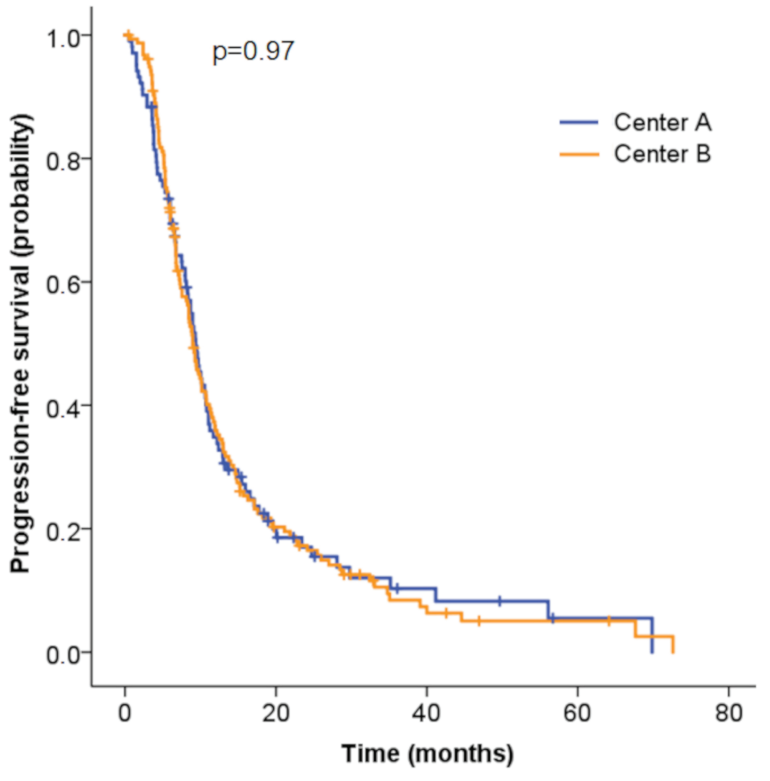

$\begin{array}{lccccccc}\text { Months } & \mathbf{0} & \mathbf{1 0} & \mathbf{2 0} & \mathbf{3 0} & \mathbf{4 0} & \mathbf{5 0} & \mathbf{6 0} \\ \text { Center A } & 103 & 43 & 15 & 7 & 5 & 3 & 1 \\ \text { Center B } & 156 & 63 & 27 & 14 & 6 & 3 & 3\end{array}$

FIG. 2. Kaplan-Meier curve for PFS between center $A$ and center $B$, showing no difference in PFS between the two centers $(p=0.97)$. Hash marks indicate censored cases.

for center B patients. The 6-month estimated survival rate was $40.5 \%$ for patients at center A compared with $55 \%$ at center $\mathrm{B}$, while at 12 months the rates were $11.3 \%$ vs $32.5 \%$, respectively. PFS did not differ between the two cohorts (center A: 9.4 months, 95\% CI 8.1-10.7 months vs center B: 9.0 months, 95\% CI 7.9-10.1 months; $\mathrm{p}=0.97$ ) (Fig. 2).

Since we observed no difference between the two cohorts with respect to well-established prognostic factors, we developed a multivariate Cox proportional hazards model to further examine the effects of the treatment centers on survival differences, with disease progression as time zero (Table 3). The rate of survival after progression in center B was $60 \%$ higher at any time than the rate of survival in center A (HR 1.59, 95\% CI 1.17-2.15). When second-line treatment ("yes/no") was added to the model, the treating center was no longer significant (HR 0.97, 95\% CI $0.62-1.50 ; p=0.88)$, indicating that the effect of the treatment center was due to the treatment strategy favored and not other unexplored differences in care quality.

\section{Discussion}

In this comparative cohort study of patients with newly diagnosed primary GBMs, a significant survival benefit was observed for patients treated at a hospital advocating second-line, tumor-specific treatment recommended by an interdisciplinary tumor board, as opposed to patients treated at a hospital with a more conservative approach favoring supportive care at recurrence, decided by the treating physician.
TABLE 3. Cox proportional hazards model: multivariate regression analysis of factors related to SaP

\begin{tabular}{lccr}
\hline \multicolumn{1}{c}{ Factor } & HR & \multicolumn{1}{c}{$95 \% \mathrm{Cl}$} & $\mathrm{p}$ Value \\
\hline Treatment center & 1.59 & $1.17-2.15$ & 0.002 \\
\hline KPS score at progression & 0.95 & $0.94-0.97$ & $<0.001$ \\
\hline Age & 1.04 & $1.03-1.05$ & $<0.001$ \\
\hline
\end{tabular}

Currently, no standard of care is established for recurrent or progressive GBM. Studies comparing various second-line treatment strategies or studies trying to identify effective therapies are plagued by the lack of appropriate control arms or by selection bias. ${ }^{26,29}$ Furthermore, the question of whether further treatment of the recurrent or progressive disease should generally be pursued or not has never been addressed. The aggressive nature of the disease, the need for the patients and their relatives to "not give up," and strong but very diverse regional treatment traditions make it very hard to conduct a relevant randomized study.

Our study takes advantage of the natural differences that result from discrepant management strategies of recurrent GBM in two centers that are very similar regarding the volume of treated patients and the first-line treatment strategies but vary considerably regarding patient management and approach upon disease progression. Center $\mathrm{B}$ has an interdisciplinary tumor board that meets once a week, where over $95 \%$ of central nervous system tumors are discussed. In Greece, the infrastructure of the healthcare system often necessitates that radiation therapy and chemotherapy take place in separate oncological hospitals, making it difficult to establish regular tumor boards. The physician responsible for deciding on further treatment remains the neurosurgeon or sometimes the oncologist. After recurrence, in center A, patients are usually offered supportive treatment and in those cases in which additional therapy is offered, it is almost always monotherapy. In contrast, in center B, patients are usually treated further, after taking into account factors such as age, performance status, timing of previous treatments, and so on. This difference in treatment philosophies allows one to study the effect of second-line treatment in contrast to supportive treatment alone. Such an effect cannot be distilled from studies that compare patients who received tumor-specific therapy against those who did not, because this separation is usually based on particular, although ill-defined, criteria. For example, it has already been shown that younger patients with a good KPS score and long treatment-free intervals are more likely to receive further treatment. ${ }^{8,11,21}$ A comparison of data published before and after the implementation of the Stupp protocol is also problematic due to the positive effect of temozolomide at primary treatment. ${ }^{6,14}$ Since some patients were treated in both centers contrary to the usual local treatment strategy, the observed difference in OS is probably an underestimation of the true benefit of second-line treatment compared to supportive care alone.

We tried to minimize discrepancies in patient selection by excluding biopsies or surgeries where the primary goal was just relieving intracranial pressure and not complete 
resection of the contrast-enhancing tumor. This allows for evaluation of a homogeneous patient population and controls for significant bias. Additionally, the fact that the PFS was very similar for both patient groups and the fact that the two cohorts were also similar with respect to baseline characteristics suggest similar resection rates for both cohorts.

The focus of this study was not to identify the optimal treatment modality for GBM progression. The focus was the comparison of two fundamentally different treatment strategies once the tumor progressed. Although center B more frequently treated GBM recurrence(s), the treatment modalities offered were highly heterogeneous. This can of course be explained by the fact that each decision was individualized, based on nonstandardized criteria such as age, previous treatments, current tumor burden, clinical condition, wish of the patient, and so on. Another important reason is the fact that as patients were treated over the years, experience was gained and beliefs were validated or rejected from accumulating evidence. For example, although the effect of repeat surgery remains unclear, a number of retrospective studies suggested a survival benefit from reoperation. ${ }^{1,10,13}$ Post hoc analysis of the prospective DIRECTOR trial showed that complete resection of the tumor was associated with improved survival for recurrent GBM. ${ }^{24}$ In our study, of the patients who had their progression treated, $55 \%$ at center $\mathrm{A}$ and $59 \%$ at center B underwent reoperation, which reflects the belief that repeat surgery offers a therapeutic effect, in addition to the benefit of collecting tumor tissue. Regarding repeat irradiation, it was offered more frequently and more generously in center B. This is due to not only published data reporting on the safety of this treatment but also accumulating in-house experience. ${ }^{3,17}$ The combination of lomustine and bevacizumab was a frequent option in center B, particularly after the results of the BELOB phase II randomized trial. However, after these promising results could not be confirmed in the EORTC 26101 trial and after bevacizumab failed to acquire approval in Germany, the use of bevacizumab, either alone or in combination with another agent, is restricted to patients for whom other options have failed. ${ }^{2,25}$

Another important difference between the two centers is implementing various combinations of treatment modalities as opposed to monotherapy. The majority $(75 \%)$ of patients treated in center A received only a single modality (usually surgery), while $64 \%$ of patients in center $B$ were treated with a combination of various modalities. This reflects the belief at center B that patients with GBM progression should receive maximal treatment, but it also mirrors the uncertainty regarding which therapy works best and with what combination.

The effect of the tumor board itself also needs to be addressed. Although an interdisciplinary setting by no means corresponds to "therapy" and of course it cannot, and should not, be quantified as such, such a collaborative approach allows for a much faster diffusion of current knowledge and experience. This in turn translates into quicker implementation of current treatments and facilitates setting up combinations of treatment modalities.

Our study has several limitations, the most important being its retrospective nature. We observed that patients in center A were less strict in adhering to the 3-month control intervals, which could lead to an overestimation of the PFS and could account for the nonsignificant difference of 0.4 months in favor of center A. In contrast, survival after progression assesses death as endpoint, which does not allow for bias other than death due to non-tumor-related causes.

Another issue that becomes very relevant when discussing repeated treatment is the quality of life (QOL) $1{ }^{19}$ While repeat treatment may prolong survival, it may do so at the cost of lowering QOL, especially when treating subsequent recurrences. Since no QOL data were collected, no conclusion can be drawn on this subject.

The noncentralized review of the tissue samples and imaging studies is a further source of bias. Although in both centers diagnosis was established by an experienced neuropathologist, we cannot exclude diagnostic errors, particularly the inclusion of cases of secondary GBMs. However, although molecular determination of IDH mutations was performed selectively, we saw no difference in the number of $I D H$-mutated GBMs between the two groups; since the PFS did not differ significantly, we believe that contamination of our cohorts with falsely diagnosed tumors was equally low. Furthermore, data on the methylation status of the $O^{6}$-methylguanine-DNA methyltransferase (MGMT) promoter was only available for German patients. Although we cannot be sure that the distribution of MGMT-positive and MGMT-negative patients was similar, no data exist to suggest a discrepant ratio of MGMT-positive to MGMT-negative patients between various geographical regions.

\section{Conclusions}

Treatment strategy favoring second-line treatment over minimal treatment or supportive care for GBM recurrence or progression is associated with significantly better survival after progression.

\section{References}

1. Bloch O, Han SJ, Cha S, Sun MZ, Aghi MK, McDermott $\mathrm{MW}$, et al: Impact of extent of resection for recurrent glioblastoma on overall survival: clinical article. J Neurosurg 117:1032-1038, 2012

2. Chinot OL, Wick W, Mason W, Henriksson R, Saran F, Nishikawa R, et al: Bevacizumab plus radiotherapy-temozolomide for newly diagnosed glioblastoma. N Engl J Med 370:709-722, 2014

3. Combs SE, Debus J, Schulz-Ertner D: Radiotherapeutic alternatives for previously irradiated recurrent gliomas. BMC Cancer 7:167, 2007

4. Combs SE, Edler L, Rausch R, Welzel T, Wick W, Debus J: Generation and validation of a prognostic score to predict outcome after re-irradiation of recurrent glioma. Acta Oncol 52:147-152, 2013

5. Delgado-Fernandez J, Garcia-Pallero MA, Blasco G, Penanes JR, Gil-Simoes R, Pulido P, et al: Usefulness of reintervention in recurrent glioblastoma: an indispensable weapon for increasing survival. World Neurosurg 108:610-617, 2017

6. Diebold G, Ducray F, Henaine AM, Frappaz D, Guyotat J, Cartalat-Carel S, et al: Management of glioblastoma: comparison of clinical practices and cost-effectiveness in two cohorts of patients (2008 versus 2004) diagnosed in a French university hospital. J Clin Pharm Ther 39:642-648, 2014

7. Dincoglan F, Beyzadeoglu M, Sager O, Demiral S, Gamsiz 
H, Uysal B, et al: Management of patients with recurrent glioblastoma using hypofractionated stereotactic radiotherapy. Tumori 101:179-184, 2015

8. Fietkau R, Putz F, Lahmer G, Semrau S, Buslei R: Can MGMT promoter methylation status be used as a prognostic and predictive marker for glioblastoma multiforme at the present time? A word of caution. Strahlenther Onkol 189:993-995, 2013

9. Fine HA, Dear KB, Loeffler JS, Black PM, Canellos GP: Meta-analysis of radiation therapy with and without adjuvant chemotherapy for malignant gliomas in adults. Cancer 71:2585-2597, 1993

10. Guyotat J, Signorelli F, Frappaz D, Madarassy G, Ricci AC, Bret P: Is reoperation for recurrence of glioblastoma justified? Oncol Rep 7:899-904, 2000

11. Kim HR, Kim KH, Kong DS, Seol HJ, Nam DH, Lim DH, et al: Outcome of salvage treatment for recurrent glioblastoma. J Clin Neurosci 22:468-473, 2015

12. Louis DN, Ohgaki H, Wiestler OD, Cavenee WK, Burger PC, Jouvet A, et al: The 2007 WHO classification of tumours of the central nervous system. Acta Neuropathol 114:97-109, 2007

13. McNamara MG, Lwin Z, Jiang H, Templeton AJ, Zadeh G, Bernstein M, et al: Factors impacting survival following second surgery in patients with glioblastoma in the temozolomide treatment era, incorporating neutrophil/lymphocyte ratio and time to first progression. J Neurooncol 117:147-152, 2014

14. Nava F, Tramacere I, Fittipaldo A, Bruzzone MG, Dimeco F, Fariselli L, et al: Survival effect of first- and second-line treatments for patients with primary glioblastoma: a cohort study from a prospective registry, 1997-2010. Neuro Oncol 16:719-727, 2014

15. Parakh S, Thursfield V, Cher L, Dally M, Drummond K, Murphy M, et al: Recurrent glioblastoma: Current patterns of care in an Australian population. J Clin Neurosci 24:78-82, 2016

16. Park JK, Hodges T, Arko L, Shen M, Dello Iacono D, McNabb A, et al: Scale to predict survival after surgery for recurrent glioblastoma multiforme. J Clin Oncol 28:3838 3843, 2010

17. Ryu S, Buatti JM, Morris A, Kalkanis SN, Ryken TC, Olson $\mathrm{JJ}$ : The role of radiotherapy in the management of progressive glioblastoma: a systematic review and evidence-based clinical practice guideline. J Neurooncol 118:489-499, 2014

18. Seystahl K, Gramatzki D, Roth P, Weller M: Pharmacotherapies for the treatment of glioblastoma-current evidence and perspectives. Expert Opin Pharmacother 17:1259-1270, 2016

19. Signorovitch J, Li N, Ohashi E, Dastani H, Shaw J, Orsini L: Overall survival (OS), quality of life (QOL), and neurocognitive function (NF) in recurrent glioblastoma multiforme (GBM): a systematic literature review. Value Health 18:A433, 2015 (Abstract)

20. Steffens R, Semrau S, Lahmer G, Putz F, Lettmaier S, Eyüpoglu I, et al: Recurrent glioblastoma: who receives tumor specific treatment and how often? J Neurooncol 128:85-92, 2016

21. Stupp R, Hegi ME, Mason WP, van den Bent MJ, Taphoorn MJ, Janzer RC, et al: Effects of radiotherapy with concomitant and adjuvant temozolomide versus radiotherapy alone on survival in glioblastoma in a randomised phase III study: 5-year analysis of the EORTC-NCIC trial. Lancet Oncol 10:459-466, 2009

22. Stupp R, Mason WP, van den Bent MJ, Weller M, Fisher B, Taphoorn MJ, et al: Radiotherapy plus concomitant and adjuvant temozolomide for glioblastoma. N Engl J Med 352:987-996, 2005
23. Stupp R, Taillibert S, Kanner AA, Kesari S, Steinberg DM, Toms SA, et al: Maintenance therapy with tumor-treating fields plus temozolomide vs temozolomide alone for glioblastoma: a randomized clinical trial. JAMA 314:2535-2543, 2015

24. Suchorska B, Weller M, Tabatabai G, Senft C, Hau P, Sabel $\mathrm{MC}$, et al: Complete resection of contrast-enhancing tumor volume is associated with improved survival in recurrent glioblastoma-results from the DIRECTOR trial. Neuro Oncol 18:549-556, 2016

25. Taal W, Oosterkamp HM, Walenkamp AM, Dubbink HJ, Beerepoot LV, Hanse MC, et al: Single-agent bevacizumab or lomustine versus a combination of bevacizumab plus lomustine in patients with recurrent glioblastoma (BELOB trial): a randomised controlled phase 2 trial. Lancet Oncol 15:943-953, 2014

26. Weller M, Cloughesy T, Perry JR, Wick W: Standards of care for treatment of recurrent glioblastoma-are we there yet? Neuro Oncol 15:4-27, 2013

27. Weller M, van den Bent M, Tonn JC, Stupp R, Preusser M, Cohen-Jonathan-Moyal E, et al: European Association for Neuro-Oncology (EANO) guideline on the diagnosis and treatment of adult astrocytic and oligodendroglial gliomas. Lancet Oncol 18:e315-e329, 2017

28. Wen PY, Macdonald DR, Reardon DA, Cloughesy TF, Sorenson AG, Galanis E, et al: Updated response assessment criteria for high-grade gliomas: response assessment in neurooncology working group. J Clin Oncol 28:1963-1972, 2010

29. Woernle CM, Péus D, Hofer S, Rushing EJ, Held U, Bozinov $\mathrm{O}$, et al: Efficacy of surgery and further treatment of progressive glioblastoma. World Neurosurg 84:301-307, 2015

\section{Disclosures}

The authors report no conflict of interest concerning the materials or methods used in this study or the findings specified in this paper.

\section{Author Contributions}

Conception and design: Stavrinou, Stranjalis. Acquisition of data: Kalyvas, Hamisch, Katsigiannis, Kabbasch. Analysis and interpretation of data: Stavrinou, Hamisch, Kabbasch. Drafting the article: Stavrinou, Kalyvas, Katsigiannis. Critically revising the article: Grau, Galldiks, Timmer, Goldbrunner, Stranjalis. Approved the final version of the manuscript on behalf of all authors: Stavrinou. Statistical analysis: Stavrinou, Timmer. Administrative/technical/material support: Goldbrunner, Stranjalis. Study supervision: Stavrinou, Stranjalis.

\section{Supplemental Information}

\section{Previous Presentations}

Portions of this work were presented in oral form at the 68th Annual Meeting of the German Society of Neurosurgery (DGNC), May 14-17, 2017, in Magdeburg, Germany, and at the 31st Annual Meeting of the Greek Society of Neurosurgery (GSNS), July 15-17, 2017, in Ioannina, Greece, where it won the first research prize. Portions of this work were presented in poster form at the 5th Quadrennial Meeting of the World Federation of Neuro-Oncology Societies, May 4-7, 2017, in Zürich, Switzerland.

\section{Correspondence}

Pantelis Stavrinou: University Hospital of Cologne, Germany. pantelis.stavrinou@uk-koeln.de. 